Note that $S_{i^{2}+1} T$ is of period 7 ; while

$$
B A^{3}: \quad z^{\prime}=\frac{z+i+1}{z}
$$

is of period 9 ; also, as a check on (27), that

$$
D C^{5} D C^{3}: \quad z^{\prime}=\frac{z+i^{2}}{i z+1} .
$$

The University of Chicago,

October 19, 1902.

\title{
GENERATIONAL RELATIONS DEFINING THE ABSTRACT SIMPLE GROUP OF ORDER 660.
}

BY PROFESSOR L. E. DICKSON.

(Read before the American Mathematical Society, October 25, 1902.)

THE abstract group of order 660 , simply isomorphic to the group of linear fractional substitutions of determinant unity taken modulo 11, may be generated by two operators $S$ and $T$ subject to the following relations: ${ }^{*}$

$$
S^{11}=I, \quad T^{2}=I, \quad(S T)^{3}=I,
$$

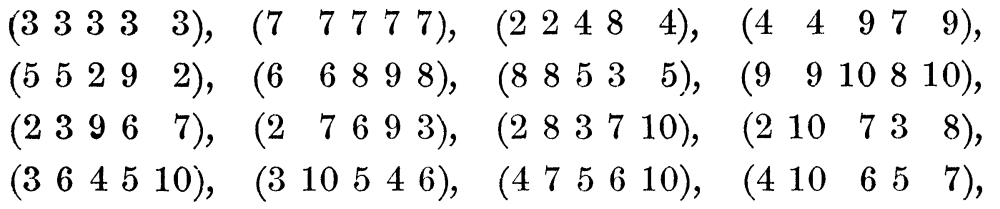
where the symbol $(a b c d e)$ denotes the relation

$$
S^{a} T S^{b} T S^{c} T S^{d} T S^{e} T=I .
$$

These relations are very redundant. We proceed to reduce

* For a proof of the general theorem due to Moore, see Dickson, Linear Groups, \& 278, Corollary. When the field is of order a prime $p$, we may set $S_{1}=S, S_{t}=S^{t}$. By $\xi 279$, we need retain only the 16 relations $(a, b, c, d, e)$ given above, since the others follow by a cyclic permutation of $a, b, c, d, e$. It may be shown that $S^{2} T, S^{3} T, S^{4} T, S^{5} T, S^{6} T, S^{7} T, S^{8} T, S^{9} T$ have the respective periods $12,5,5,6,6,5,5,12$, results not used here. 
the nineteen to five simple relations. For use in this reduction we note that from (1) follows

$$
T S T=S^{-1} T S^{-1}, \quad T S^{-1} T=S T S,
$$

(3) $\quad T S^{2} T=S^{-1} T S^{-2} T S^{-1}, \quad T S^{-2} T=S T S^{2} T S$.

Applying to (2 24484 ) relation $\left(3_{1}\right)$, we get

$$
\begin{gathered}
S T S^{-2} T S^{3} T S^{8} T S^{4} T=I . \\
S^{3} T S^{8} T=T S^{2} T S^{-1} T S^{7}=T S^{3} T S^{8},
\end{gathered}
$$

in view of $\left(2_{2}\right)$. Hence (2 24484 ) may be replaced by

$$
\left(S^{3} T S^{8} T\right)^{2}=I \text {. }
$$

By (2 $\left.24 \begin{array}{lll}4 & 4\end{array}\right)$ we have

$$
S^{7} T S^{9} T=T S^{8} T S^{4} T S^{2} .
$$

Substituting this value in (44979), we get

$$
\left(S^{4} T S^{6} T\right)^{2}=I \text {. }
$$

To (5 $\left.5 \begin{array}{lll}5 & 9 & 2\end{array}\right)$ we apply $\left(3_{1}\right)$ twice in succession. Transforming the result by $S$, we obtain (4 49979 ).

In (6 $\left.6 \begin{array}{lll}6 & 8 & 9\end{array}\right)$ we replace $S^{6} T S^{6} T$ by $T S^{2} T S^{9} T S^{2}$ given

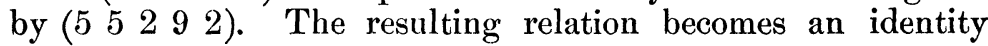
upon applying $\left(2_{2}\right)$ twice.

Transforming ( 885535$)$ by $S^{7}$ and twice replacing $T S^{5} T S^{7}$ by its inverse $S^{4} T S^{6} T$, in view of (5), we obtain $(S T)^{3}=I$.

In (9 910810$)$ we apply $\left(2_{2}\right)$ three times in succession and obtain an identity.

Transforming (2 39967 ) by $S^{5}$ and replacing $S^{8} T S^{3} T$ and $T S^{7} T S^{5}$ by their inverses, given by (4) and (5), we get

$$
T S^{8} T S T S T S^{4} T=T S^{7}(S T)^{3} S^{4} T=I .
$$

Transforming (2 76993 ) by $S^{8}$ and replacing $S^{5} T S^{7} T$ and $T S^{3} T S^{8}$ by their inverses, we get

$$
T S^{4} T S T S T S^{8} T=T S^{3}(S T)^{3} S^{8} T=I .
$$

To (2 $\left.8 \begin{array}{llll}2 & 3 & 7 & 10\end{array}\right)$ we apply $\left(2_{2}\right)$ and transform the result by $S^{-1}$. There results relation (4).

Applying $\left(2_{2}\right)$ to (2 10738 ), we get (4).

In $\left(\begin{array}{lllll}3 & 6 & 4 & 5 & 10\end{array}\right)$ we replace $T S^{6} T S^{4}$ by its inverse and get

$$
\left(S^{-1} T\right)^{3}=I \text {. }
$$


Similarly, (3 10546 ), (4 756 10), and (4 1065 7) follow by applying first (5) and then (1).

Transforming (3 33333 ) by $S^{6}$ and replacing $S^{8} T S^{3} T$ by its inverse $T S^{8} T S^{3}$, given by (4), we get

$$
T S^{8} T S^{6} T S^{3} T S^{3} T S^{6}=I,
$$

a relation following by taking the inverse of ( 885535 ).

Transforming (7 7777 ) by $S^{2}$ and replacing $S^{5} T S^{7} T$ by its inverse $T S^{4} T S^{6}$, given by $(5)$, we get

$$
T S^{4} T S^{2} T S^{7} T S^{7} T S^{2}=I,
$$

a relation following by taking the inverse of (4 4979 ).

Hence the simple group of order 660 is generated by two operators $S$ and $T$ subject to relations (1), (4), and (5).

The University of Chicago,

$$
\text { October 22, } 1902 .
$$

\section{THE CARLSBAD MEETING OF THE DEUTSCHE MATHEMATIKER-VEREINIGUNG, SEPTEMBER, 1902.}

THE annual meeting of the Deutsche Mathematiker-Vereinigung was held at Carlsbad, September 21-26, forming as usual a part of the Versammlung Deutscher Naturforscher und Aertze. At the business meeting of the Society, committees were appointed to make arrangements for the international mathematical congress which will be held at Heidelberg in August, 1904. A committee was also appointed to consider the feasibility of forming at some point in Germany a very complete collection of mathematical literature. Professor Gutzmer resigned from the position of secretary of the society, and Professor Krazer was elected his successor. The meeting of the Society will be held next year at Cassel.

The following papers were read at the meeting:

Professor Czuber, Vienna: "A theorem in the theory of errors, and its application."

Professor GRÜNWALD, Prague: "Derivatives with variable index as analytic functions of the latter, and a related functional operation." 